\title{
Infrared identification of IGR J09026-4812 as a Seyfert 1 galaxy ${ }^{\star}$
}

\author{
J. A. Zurita Heras ${ }^{1}$, S. Chaty ${ }^{1}$, and J. A. Tomsick ${ }^{2}$
}

\author{
1 Laboratoire AIM, CEA/DSM-CNRS-Université Paris Diderot, IRFU/Service d'Astrophysique, 91191 Gif-sur-Yvette, France \\ e-mail: [juan-antonio.zurita-heras; sylvain.chaty]@cea.fr \\ 2 Space Sciences Laboratory, 7 Gauss Way, University of California, Berkeley, CA 94720-7450, USA \\ e-mail: jtomsick@ssl.berkeley.edu
}

Received 21 April 2009 / Accepted 4 May 2009

ABSTRACT

\begin{abstract}
Context. IGR J09026-4812 was discovered by INTEGRAL in 2006 as a new hard X-ray source. Thereafter, an observation with Chandra pinpointed a single X-ray source within the ISGRI error circle, showing a hard spectrum, and improving its high-energy localisation to a subarcsecond accuracy. Thus, the X-ray source was associated with the infrared counterpart 2MASS J09023731-4813339 whose $J H K_{\mathrm{S}}$ photometry indicated a highly reddened source. The high-energy properties and the counterpart photometry suggested a high-mass X-ray binary with a main sequence companion star located 6.3-8.1 kpc away and with a $0.3-10 \mathrm{keV}$ luminosity of $8_{-1}^{+13} \times 10^{34} \mathrm{erg} \mathrm{s}^{-1}$.

Aims. New optical and infrared observations were needed to confirm the counterpart and to reveal the nature of IGR J09026-4812.

Methods. We performed optical and near infrared observations on the counterpart 2MASS J09023731-4813339 with the ESO/NTT telescope on March 2007. We carried out photometry and spectroscopy in near infrared wavelengths and photometry in optical wavelengths.

Results. The accurate astrometry at both optical and near infrared wavelengths confirmed 2MASS J09023731-4813339 as the counterpart of IGR J09026-4812. However, the near infrared images show that the source is extended, thus excluding the possibility of a Galactic compact source. The source spectrum shows three main emission lines identified as the HeI $\lambda 1.0830 \mu \mathrm{m}$ line, and the $\mathrm{HI} \mathrm{Pa} \beta$ and $\mathrm{Pa} \alpha$ lines, typical of galaxies with an active galactic nucleus. The broadness of these lines reached values as large as $4000 \mathrm{~km} \mathrm{~s}^{-1}$, suggesting a type 1 Seyfert galaxy. The redshift of the source is $z=0.0391 \pm 0.0004$. Thus, the near infrared photometry and spectroscopy allowed us to classify IGR J09026-4812 as a type 1 Seyfert galaxy.
\end{abstract}

Key words. galaxies: Seyfert - infrared: galaxies - X-rays: individual: IGR J09026-4812

\section{Introduction}

The ESA high-energy space mission INTEGRAL (Winkler et al. 2003) performed a deep survey of our Galaxy, allowing the discovery of many new hard X-ray sources. This was achieved using the coded-mask imager IBIS/ISGRI $(15 \mathrm{keV}-1 \mathrm{MeV}$, field of view of $29^{\circ} \times 29^{\circ}$, angular resolution of $12^{\prime}$; Ubertini et al. 2003; Lebrun et al. 2003). A 20-100 keV hard X-ray emission such as that observed by ISGRI has been regularly reported (Bird et al. 2006, 2007; Bodaghee et al. 2007). The unclassified source IGR J09026-4812 was first reported in the 2nd ISGRI catalogue as a new hard X-ray source (Bird et al. 2006). Its position was RA $(2000)=135.638$ and $\mathrm{Dec}=$ $-48.196\left(1.1^{\prime}\right.$ at $68 \%$ confidence level). The average $20-40$ and $40-100 \mathrm{keV}$ fluxes were $0.9 \pm 0.1$ and $0.9 \pm 0.2 \mathrm{mCrab}$, respectively, for an effective exposure of $1.35 \mathrm{Ms}$. These values only underwent a slight change in the 3rd ISGRI catalogue (Bird et al. 2007): RA $(2000)=135^{\circ} .668$, Dec $=-48^{\circ} .216$ ( $2.3^{\prime}$ at $90 \%$ confidence level), $F_{20-40 \mathrm{keV}}=1.3 \pm 0.1 \mathrm{mCrab}$ and $F_{40-100 \mathrm{keV}}=1.4 \pm 0.2 \mathrm{mCrab}$, for an effective exposure of $1.5 \mathrm{Ms}$. The source IGR J09026-4812 was tentatively associated with a faint ROSAT counterpart located $2.5^{\prime}$ away from the ISGRI position at RA $(2000)=09^{\mathrm{h}} 02^{\mathrm{m}} 38.4^{\mathrm{s}}$ and

^ Based on observations made with ESO Telescopes at the La Silla Observatory under programme ID 078.D-0268(B).
Dec $=-48^{\circ} 14^{\prime} 08.0^{\prime \prime}\left(20^{\prime \prime}\right)$ that showed an average count rate of $(2.7 \pm 0.9) \times 10^{-2}$ counts s $^{-1}($ Stephen et al. 2006).

Tomsick et al. (2008) reported a 5 ks Chandra observation performed on Feb. 5, 2007 that showed a single X-ray source within the ISGRI error circle located at RA (2000) = $09^{\mathrm{h}} 02^{\mathrm{m}} 37.33^{\mathrm{s}}$ and Dec $=-48^{\circ} 13^{\prime} 34.1^{\prime \prime}\left(0.6^{\prime \prime}\right.$ at $90 \%$ confidence level). This position is located outside the ROSAT error circle, excluding the soft X-ray counterpart suggested by Stephen et al. (2006). The $0.3-10 \mathrm{keV}$ spectrum was fitted with an absorbed power law model: $N_{\mathrm{H}}=\left(1.9_{-0.4}^{+0.6}\right) \times 10^{22} \mathrm{~cm}^{-2}, \Gamma=1.1_{-0.3}^{+0.5}(90 \%$ confidence errors) and an unabsorbed $0.3-10 \mathrm{keV}$ flux of $1.3 \times$ $10^{-11} \mathrm{erg} \mathrm{cm}^{-2} \mathrm{~s}^{-1}$. They also showed that the source was not intrinsically absorbed when comparing the fitted $N_{\mathrm{H}}$ to the atomic and molecular hydrogen column densities through our Galaxy. They suggested the source 2MASS J09023731-4813339 as the infrared (IR) counterpart since this source was located $0.4^{\prime \prime}$ away from the Chandra position. Its IR magnitudes were $J=$ $15.57 \pm 0.08, H=13.86 \pm 0.07$, and $K_{\mathrm{S}}=12.69 \pm 0.04$. Due to its $K_{\mathrm{S}}$ brightness, high extinction, and hard power law, Tomsick et al. (2008) suggested that IGR J09026-4812 was a new highmass X-ray binary (HMXB) with a main sequence companion star.

We performed optical and near infrared (NIR) observations on IGR J09026-4812 in order to determine its nature. We describe the observations and the data reduction in Sect. 2. We 
Table 1. NIR and optical photometry of the single counterpart.

\begin{tabular}{cccc}
\hline \hline Date & \multicolumn{3}{c}{ 2007-03-05T01:27-02:00 } \\
Filters & $J$ & $H$ & $K_{\mathrm{S}}$ \\
\hline Mag. & $15.2 \pm 0.1$ & $13.66 \pm 0.03$ & $12.3 \pm 0.2$ \\
$Z_{\mathrm{p}}$ & $2.0 \pm 0.1$ & $2.12 \pm 0.03$ & $2.9 \pm 0.2$ \\
$A M^{\dagger}$ & 1.10 & 1.09 & 1.08 \\
SMA $\left.^{\prime \prime}\right]$ & 3.3 & 4.0 & 4.9 \\
PA $\left.{ }^{\circ}\right]$ & $-50 \pm 11$ & $-60 \pm 3$ & $-51 \pm 2$ \\
Ecc. & $0.51 \pm 0.09$ & $0.75 \pm 0.03$ & $0.78 \pm 0.02$ \\
\hline Date & \multicolumn{3}{c}{$2007-03-10 \mathrm{~T} 00: 21-00: 41$} \\
Filters & $J$ & $H$ & $K_{\mathrm{S}}$ \\
\hline Mag. & $15.24 \pm 0.02$ & $13.63 \pm 0.02$ & $12.53 \pm 0.08$ \\
$Z_{\mathrm{p}}$ & $1.929 \pm 0.002$ & $2.07 \pm 0.02$ & $2.62 \pm 0.07$ \\
$A M^{\dagger}$ & 1.20 & 1.18 & 1.16 \\
SMA[ $\left.{ }^{\circ}\right]$ & 3.3 & 4.0 & 4.9 \\
PA[ $\left.{ }^{\circ}\right]$ & $-64 \pm 7$ & $-62 \pm 3$ & $-50 \pm 2$ \\
Ecc. & $0.68 \pm 0.06$ & $0.71 \pm 0.03$ & $0.74 \pm 0.02$ \\
\hline Date & \multicolumn{2}{c}{$2007-03-14 T 00: 26-00: 36$} \\
Filters & $V$ & $R$ & $I$ \\
\hline Mag. & $>23.3 \pm 0.2$ & $20.8 \pm 0.2$ & $19.3 \pm 0.2$ \\
$Z_{\mathrm{p}}$ & $-0.50 \pm 0.01$ & $-0.39 \pm 0.01$ & $0.47 \pm 0.02$ \\
$A M$ & 1.14 & 1.14 & 1.13 \\
ext & 0.13 & 0.09 & 0.05 \\
\hline \multicolumn{3}{c}{}
\end{tabular}

- Total NIR magnitudes were extracted within ellipses centred on the source and defined with the semi-major axis (SMA), the position angle (PA, relative to west axis and counter clockwise), and the eccentricity (Ecc.); ${ }^{\dagger}$ The NIR extinction parameters were fixed to $J H K_{\mathrm{S}}=$ $0.07,0.03,0.03$ with an error of $\Delta e x t / e x t=0.2$.

present the results and discuss the nature of this source in Sect. 3, and conclude in Sect. 4.

\section{Observations and data analysis}

The observations were carried out with the ESO $3.6 \mathrm{~m} \mathrm{New}$ Technology Telescope (NTT) at La Silla Observatory, Chile, as part of the program 078.D-0268(B) through service mode. The optical and NIR observations were obtained with the imager SUSI-2 (350-900 $\mathrm{nm})$ and the spectro-imager SOFI (0.9-2.4 $\mu \mathrm{m})$, respectively. Both instruments are installed at the same Nasmyth focus of the NTT. Astrometry, photometry and spectroscopy were achieved during these observations.

\subsection{NIR observations}

NIR photometry in the $J, H$ and $K_{\mathrm{S}}$ bands was performed on March 5 and 10, 2007. The observations were centred on the accurate X-ray position of IGR J09026-4812. The large imaging mode was used, which implied an image pixel scale of $0.288^{\prime \prime} /$ pixel and a field of view of $4.92^{\prime} \times 4.92^{\prime}$. Nine images were taken for each filter $J, H$ and $K_{\mathrm{S}}$ with integration times of $60 \mathrm{~s}$ each. For each filter, four of the nine images were taken with a slight offset of $\sim 30^{\prime \prime}$ that allowed us to build the NIR sky in order to subtract it from the images. Two standard stars chosen from the faint NIR standard star catalogue (Persson et al. 1998) were also observed: S255-S and S262-E. Five observations per filter were performed on each standard star. The first observation was centred on the target, and the next four images were taken with an offset of $\sim 45^{\prime \prime}$ compared to the first one. The same strategy with the same standard stars was applied during the 2 nd night. The seeing conditions varied from $\sim 0.9^{\prime \prime}$ during the 1 st night to $\sim 0.5^{\prime \prime}$ during the 2 nd night.
NIR spectroscopy using both low-resolution blue and red grisms was carried out on March 5 and 12, 2007. For each filter, twelve spectra were taken with the $1.0^{\prime \prime}$ slit. Half of the spectra were taken on the source and the other half with an offset of $60^{\prime \prime}$ in order to subtract the NIR sky from the images, the jitter pattern being AAA BBB BBB AAA. The integration time was $180 \mathrm{~s}$ for each spectrum for a total observation of $4320 \mathrm{~s}$. Two spectroscopic standard stars were observed: HD62388 (spectral type (sp.T.) A0V) and Hip0842847 (sp.T. G2V) on March 5 and 12, respectively. For each standard star, four spectra in both blue and red grisms were taken, of which half were taken with an offset of $45^{\prime \prime}$.

\subsection{Optical observations}

Only optical photometry was carried out on March 14, 2007 , with the filters $U, B, V, R, I, Z$. One image per filter was obtained with a field of view of $5.5^{\prime} \times 5.5^{\prime}$ with a binning factor of 2 , which implied a pixel scale of $0.161^{\prime \prime} /$ pixel. The integration time was $60 \mathrm{~s}$ in each filter. Thirteen photometric standard stars selected in the optical standard star catalogue of Landolt (1992) were observed in the 2 fields 98 (733,1087,1102,1112,1119,1122,1124) and RU_152+RU_152(A,B,C,E,F). The integration times varied between 5 and $30 \mathrm{~s}$.

\subsection{Data reduction}

The reduction of both optical and NIR data was performed with the Image Reduction and Analysis Facility (IRAF ${ }^{1}$ ) version 2.13beta2. Data reduction was performed using standard procedures on the optical and NIR images, including crosstalk, correction of the dark current (in optical wavelengths), flatfielding and NIR sky subtraction.

We performed accurate astrometry on each image ( $U, B, V, R, I, Z, J, H, K_{\mathrm{S}}$ ) using the gaia tool from the Starlink suite and using the 2MASS catalogue for the NIR images and the USNO B1.0 catalogue for the optical images. The NIR root mean square (rms) of the astrometrical fit was always lower than $0.4^{\prime \prime}$, with the expected pixel scale in the $x, y$ axis of $-0.288 \times 0.288^{\prime \prime} /$ pixel. The optical rms of the astrometrical fit was also lower than $0.4^{\prime \prime}$, with the expected pixel scale in the $x, y$ axis of $0.161 \times 0.161^{\prime \prime} /$ pixel.

We carried out aperture photometry in a crowded field using the IRAF digiphot.daophot package. For the NIR photometry, the point sources located near IGR J09026-4812 were subtracted using the same package. Aperture photometry on the source was performed with several ellipses using the stsdas.isophot and digiphot.apphot packages. The instrumental magnitudes $m_{\text {instr }}$ were transformed into apparent magnitudes $m_{\text {app }}$ using the standard photometric relation $m_{\text {app }}=$ $m_{\text {instr }}-Z_{\mathrm{p}}-$ ext $\times A M$, where $Z_{\mathrm{p}}$ is the zero-point, ext the extinction and $A M$ the airmass. The colour term was not used in the NIR because there were not enough standard stars and not at optical wavelengths because we did not detect the sources of interest in several bands, particularly in $V$. The $Z_{\mathrm{p}}$ parameters were fitted using the photometric relation in order to match the instrumental and apparent magnitudes of the standard stars. The extinction parameters were fixed to the values given in the SOFI and SUSI- 2 calibration tables ${ }^{2}$. The magnitudes, airmass, zeropoint and extinction parameters are reported in Table 1. Images

\footnotetext{
1 IRAF is available at http: //iraf . net/

2 Retrieved at http://www . eso.org/sci/facilities/lasilla/ telescopes/ntt/index.html
} 


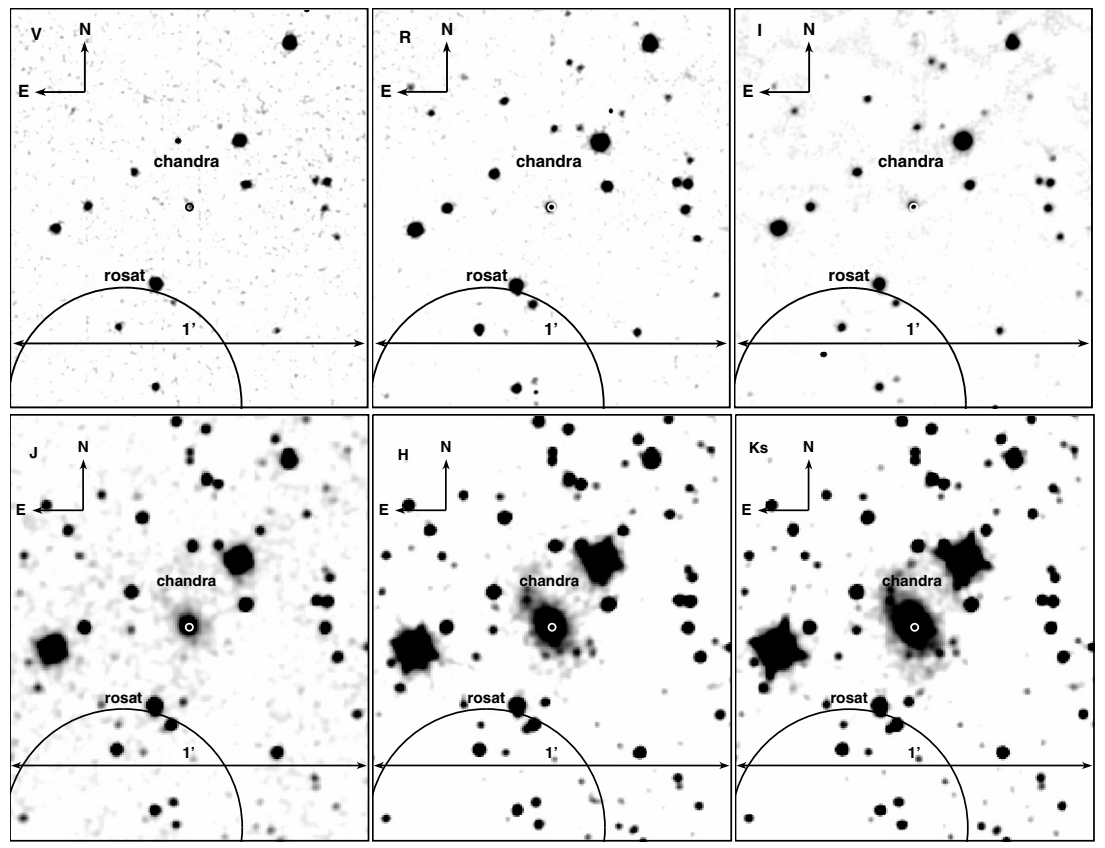

Fig. 1. Left to right, top to bottom: VRI and $J H K_{\mathrm{s}}$ images taken on March 10 (NIR) and 14 (optical), 2007.

in $V R I J H K_{\mathrm{s}}$ are shown in Fig. 1. The NIR surface brightness profiles are shown in Fig. 2.

The NIR spectra were reduced using the IRAF noao. twodspec package. Each individual image was corrected using standard procedures for the crosstalk and flat-fielding. Consecutive images with the same jitter were combined to obtain 4 output images. They were corrected for the NIR sky, subtracting images with different jitter. We extracted the source spectrum from the 4 images. We performed wavelength calibrations using a xenon arc extracted with the same parameters as for the source. The 4 spectra were combined in a single spectrum and corrected for telluric features using the spectrum of the standard star and the IRAF tool telluric. We applied this strategy for both blue and red filters and combined the blue and red spectra. We multiplied the full spectrum by a calibrated spectrum of an A0 V (or G2 V) star from the spectral library of Pickles (1998) to avoid spectral contamination from the telluric star. The output spectra were dereddened from the Galactic extinction using $E(B-V)=1.509$ (Schlegel et al. 1998). The resulting spectra are shown in Fig. 3.

\section{Results and discussion}

Only one optical and NIR candidate was located within the Chandra error circle (see Fig. 1). Our NIR astrometry confirmed the candidate 2MASS J09023731-4813339 suggested by Tomsick et al. (2008).

The high-energy properties of the source and the NIR photometry of the 2MASS source led to a tentative classification of the source as an HMXB with a main sequence star (Tomsick et al. 2008). However, this hypothesis is discarded by the NIR images as IGR J09026-4812 appears as a non-circular extended source with an extension in the north-east/south-west direction with a semi-major axis of $4.9^{\prime \prime}$ (in $K_{\mathrm{S}}$ ). The source was detected from filter $R$ to filter $K_{\mathrm{S}}$. The source appears point-like in the optical images. Since the X-ray observation was performed with Chandra, that has an angular resolution similar to the optical/NIR images, this implies that we observed two different emitting components of the system between the X-ray/optical

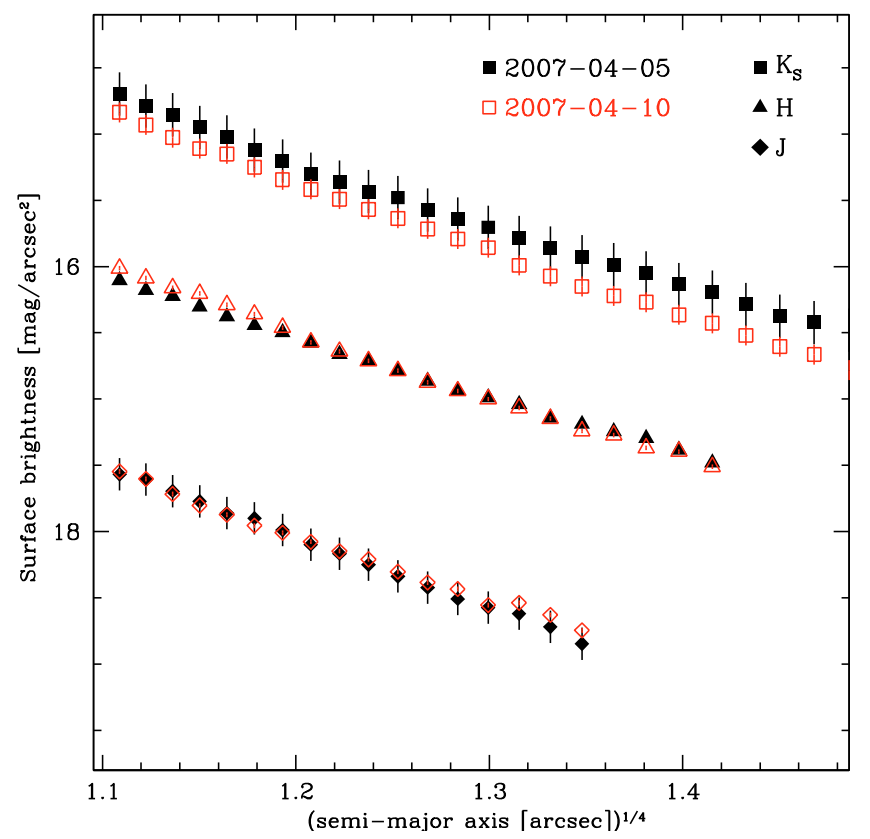

Fig. 2. NIR surface brightness profiles of IGR J09026-4812.

and the NIR. The most obvious candidate is thus a galaxy with an active galactic nucleus (AGN), plausibly a Seyfert galaxy, where the X-ray/optical radiation mainly comes from the accreting gas surrounding the supermassive black hole and the NIR radiation is mainly due to the dust present in the host galaxy (Wilkes 2004). The NIR also suggests the presence of spiral arms of the galaxy. The NIR magnitudes (reported in Table 1) do not show any variation between the two observations separated by 5 days, but indicate a strong reddening of the source with NIR color $J-K=1.97 \pm 0.08(1.93 \pm 0.13)$ mag for the March 10th (March 5th) observation, after correcting for the Galactic extinction $(E(B-V)=1.509$, Schlegel et al. 1998) and with the transformation $K-K_{\mathrm{s}}=-0.005 \times(J-K)$ (p.7, SOFI manual). Such strong reddening is mainly observed in Seyfert 1 galaxies 
Table 2. NIR spectroscopy. Emission lines are represented with the laboratory $\left(\lambda_{0}\right)$ and fitted $\left(\lambda_{\text {fit }}\right)$ wavelengths.

\begin{tabular}{|c|c|c|c|c|c|c|c|c|c|}
\hline \multirow[b]{2}{*}{ Identification } & \multirow[b]{2}{*}{$\begin{array}{c}\lambda_{0} \\
\mu \mathrm{m}\end{array}$} & \multicolumn{4}{|c|}{ March 5} & \multicolumn{4}{|c|}{ March 12} \\
\hline & & $\begin{array}{l}\lambda_{\text {fit }} \\
\mu \mathrm{m}\end{array}$ & $\begin{array}{c}\text { Flux } \\
\text { arbitrary }\end{array}$ & $\begin{array}{c}E W \\
\AA\end{array}$ & $\begin{array}{c}F W H M \\
\AA\end{array}$ & $\begin{array}{l}\lambda_{\text {fit }} \\
\mu \mathrm{m}\end{array}$ & $\begin{array}{c}\text { Flux } \\
\text { arbitrary }\end{array}$ & $\begin{array}{c}E W \\
\AA\end{array}$ & $\begin{array}{c}F W H M \\
\AA\end{array}$ \\
\hline [S III] & 0.9531 & - & - & $\begin{array}{ll}- \\
-\end{array}$ & - & $0.9911(1)$ & $2724 \pm 226$ & $-23 \pm 2$ & $35 \pm 3$ \\
\hline $\mathrm{He} \mathrm{I/Pa} \gamma$ & $1.0830 / 1.0935$ & $1.1292(6)$ & $1418 \pm 96$ & $-168 \pm 11$ & $222 \pm 18$ & $1.1293(2)$ & $19513 \pm 341$ & $-155 \pm 3$ & $182 \pm 4$ \\
\hline $\mathrm{Pa} \beta$ & 1.2820 & - & - & - & - & $1.3330(5)$ & $8621 \pm 531$ & $-59 \pm 4$ & $183 \pm 15$ \\
\hline $\operatorname{Pa} \alpha$ & 1.8751 & $1.9466(8)$ & $1120 \pm 56$ & $-170 \pm 8$ & $279 \pm 21$ & $1.9473(2)$ & $12993 \pm 167$ & $-120 \pm 1$ & $269 \pm 4$ \\
\hline
\end{tabular}

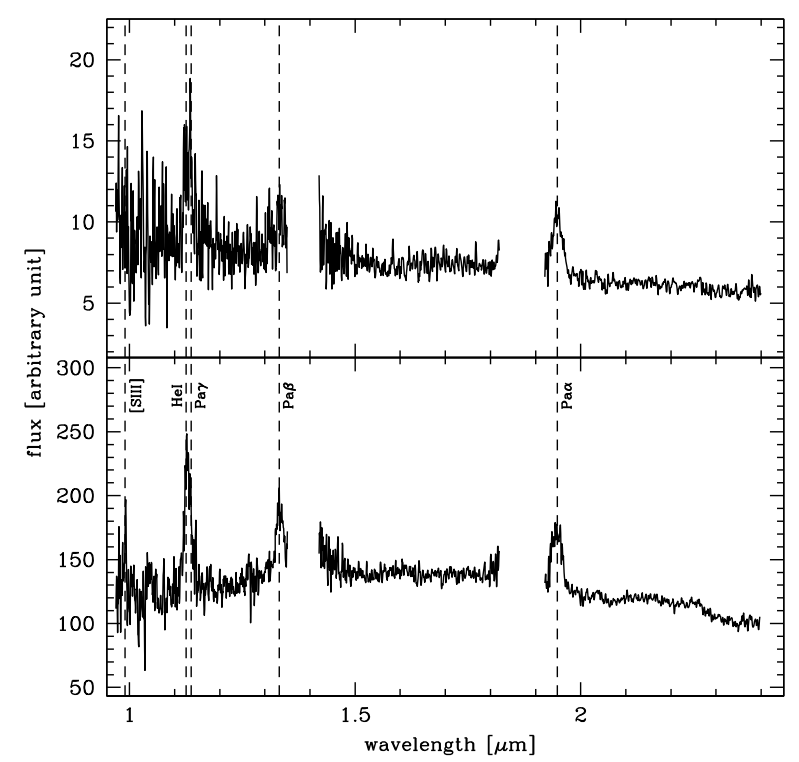

Fig. 3. NIR spectra of IGR J09026-4812 on March 5 (top) and March 12 (bottom), 2007. The dashed lines represent the identified emission lines red-shifted with $z=0.0391 \pm 0.0004$.

(Rudy et al. 1982) and was interpreted as the reprocessing of hot dust located in the broad-line region (Barvainis 1987). Mid IR observations are necessary to further study the dusty component of the Seyfert galaxy. The NIR surface brightness profiles are in good agreement with de Vaucouleurs' law $\left(S_{v} \propto R^{1 / 4}\right.$, see Fig. 2).

The emission lines observed in the two spectra (see Fig. 3, the bottom spectrum having a higher signal-to-noise ratio) are reported in Table 2 . The three main emission lines of the spectra situated at $\lambda 1.1288 \mu \mathrm{m}, \lambda 1.3323 \mu \mathrm{m}$ and $\lambda 1.9490 \mu \mathrm{m}$ were easily identified with the $\mathrm{HeI} \lambda 1.0830 \mu \mathrm{m}$ and $\mathrm{HI}(\mathrm{Pa} \beta$ and $\mathrm{Pa} \alpha)$ emission lines, once red-shifted by $\sim 0.4$. These are common emission lines in AGN, independent of the class (see the spectral atlas of AGN in the energy range $0.8-2.4 \mu \mathrm{m}$ of Riffel et al. 2006, which covers the spectral range of SOFI). These emission lines are broad with values $>4000 \mathrm{~km} \mathrm{~s}^{-1}$. Such broad emission lines are typical of type $1 \mathrm{AGN}$.

We also tried to identify other emission lines, particularly in the spectrum taken on March 12, 2007 (see Fig. 3 bottom): 1) a narrow forbidden line [S III] $\lambda 0.9531 \mu \mathrm{m}$ with $F W H M=$ $1102 \pm 94 \mathrm{~km} \mathrm{~s}^{-1}$; and 2) an HI Pa $\gamma$ line that might be blended within the HeI $\lambda 1.0830 \mu \mathrm{m}$ line. However the HeI line is always the strongest in all AGN spectra (e.g. Figs. 9-12 in Riffel et al. 2006). Using only the $\mathrm{HI} \operatorname{Pa} \beta$ and $\mathrm{Pa} \alpha$ emission lines and both spectra, we derived an average redshift of $z=0.0391 \pm 0.0004$. The line ratio of $\mathrm{Pa} \alpha / \mathrm{Pa} \beta$ gave $F(\mathrm{~Pa} \alpha) / F(\mathrm{~Pa} \beta)=1.5 \pm 0.1$. This value is slightly lower than the expected value of $1.8 \pm 0.1$ for Case B recombination when considering a typical broad-line cloud with temperatures between $(0.5-3) \times 10^{4} \mathrm{~K}$ and electron densities between $10^{8-10} \mathrm{~cm}^{-3}$. Still, this is a common feature in Seyfert galaxies (Glikman et al. 2006, and references therein).

\section{Conclusion}

We conclude that IGR J09026-4812 is a type 1 Seyfert galaxy, as demonstrated by the optical and NIR observations. The type 1 classification is in good agreement with the lack of strong absorption observed in X-rays. Its redshift is $z=0.0391 \pm 0.0004$. Butler et al. (2009) reported that the distribution of redshifts for the AGNs detected by INTEGRAL peaked at $z=0.033$ ( 0.035 for IGRs). In the current census of sources detected by INTEGRAL/ISGRI, there are 165 detected Seyfert galaxies (of which 63 are IGR sources). Discarding sources without a reported redshift, the average redshift is 0.04 (0.06 when considering only IGRs) with a variance of 0.005 (0.006 for IGR) (Beckmann et al. 2009). IGR J09026-4812 is in good agreement with previous IGR sources identified as Seyfert galaxies and more generally with the Seyfert galaxies detected by INTEGRAL.

Acknowledgements. J.A.Z.H. thanks Farid Rahoui, Volker Beckmann and Simona Soldi for useful discussions on IGR J09026-4812. J.A.Z.H. acknowledges the Swiss National Science Foundation for financial support. J.A.T. acknowledges partial support from Chandra award GO8-9055X issued by the Chandra X-ray Observatory Center, which is operated by the Smithsonian Astrophysical Observatory for and on behalf of NASA under contract NAS8-03060. This work was supported by the Centre National d'Etudes Spatiales (CNES). It is based on observations with IBIS on INTEGRAL. The authors acknowledge the use of 1) NASA's Astrophysics Data System; 2) the SIMBAD database, operated at CDS, Strasbourg, France; and 3) data products from the Two Micron All Sky Survey, which is a joint project of the University of Massachusetts and the Infrared Processing and Analysis Center/California Institute of Technology, funded by the National Aeronautics and Space Administration and the National Science Foundation.

\section{References}

Barvainis, R. 1987, ApJ, 320, 537

Beckmann, V., Soldi, S., Ricci, C., et al. 2009, A\&A, Submitted Bird, A. J., Barlow, E. J., Bassani, L., et al. 2006, ApJ, 636, 765 Bird, A. J., Malizia, A., Bazzano, A., et al. 2007, ApJS, 170, 175 Bodaghee, A., Courvoisier, T. J.-L., Rodriguez, J., et al. 2007, A\&A, 467, 585 Butler, S. C., Tomsick, J. A., Chaty, S., et al. 2009, ArXiv e-prints Glikman, E., Helfand, D. J., \& White, R. L. 2006, ApJ, 640, 579 Landolt, A. U. 1992, AJ, 104, 340

Lebrun, F., Leray, J. P., Lavocat, P., et al. 2003, A\&A, 411, L141 Persson, S. E., Murphy, D. C., Krzeminski, W., Roth, M., \& Rieke, M. J. 1998, AJ, 116, 2475

Pickles, A. J. 1998, PASP, 110, 863

Riffel, R., Rodríguez-Ardila, A., \& Pastoriza, M. G. 2006, A\&A, 457, 61

Rudy, R. J., Levan, P. D., \& Rodriguez-Espinosa, J. M. 1982, AJ, 87, 598

Schlegel, D. J., Finkbeiner, D. P., \& Davis, M. 1998, ApJ, 500, 525

Stephen, J. B., Bassani, L., Malizia, A., et al. 2006, A\&A, 445, 869

Tomsick, J. A., Chaty, S., Rodriguez, J., Walter, R., \& Kaaret, P. 2008, ApJ, 685, 1143

Ubertini, P., Lebrun, F., Di Cocco, G., et al. 2003, A\&A, 411, L131

Wilkes, B. 2004, in AGN Physics with the Sloan Digital Sky Survey, ed. G. T. Richards, \& P. B. Hall, ASP Conf. Ser., 311, 37

Winkler, C., Courvoisier, T. J.-L., Di Cocco, G., et al. 2003, A\&A, 411, L1 